\title{
The Determinants and Subsequent Effect of Self-Rated Health Status on Survival among Elderly Americans
}

\author{
Guangming Han \\ Department of Epidemiology, University of Nebraska Medical Center, Omaha, NE, USA
}

\begin{abstract}
Article Info
Article history:

Received Jan 31, 2014

Revised Apr 20, 2014

Accepted May 26, 2014

\section{Keyword:}

Self-rated health

Mortality

Chronic disease

Elderly population

ABSTRACT

The main aim of this study is to explore the patterns, determinants and subsequent mortality prediction of change in self-rated health in the elderly American population. To achieve this purpose, we constructed logistic regression models and Cox proportional hazard regression models with the complex survey dataset from the National Second Longitudinal Study of Aging (LSOA II) to calculate the odds ratios (OR)/ hazard ratios (HR) and confidence intervals (CI) of risk factors. Our results show that chronic disease condition and difficulty in daily activities are the main reasons for change in self-rated health status. Furthermore, change in self-rated health has significant impact on survival function in the elderly populations. When change in self-rated health status was considered, self-rated health was a stronger and more flexible predictor of mortality for elderly populations. These findings will provide important information to establish effective strategies for prolonging lifespan by improving self-rated health status for elderly populations.
\end{abstract}

Copyright (C) 2014 Institute of Advanced Engineering and Science. All rights reserved.

\section{Corresponding Author: \\ Guangming Han, \\ Department of Epidemiology, University of Nebraska Medical Center, Omaha, NE, USA. \\ Email: guangming.han@unmc.edu}

\section{INTRODUCTION}

Population aging is becoming an increasing important issue worldwide. In the U.S, the elder population (age 65 years and elder) is 39.6 million in 2009, an increase of 4.3 million, or 12.5\% since 1999 . That means one in every eight, or $12.9 \%$ of the population is an elder American [1]. The world's elder population is projected to triple from 516 million in 2009 to 1.53 billion in 2050 [2]. Therefore, promoting health and prolonging life are important public health issue in elder population.

Effective promotion health and prolonging life in elder population depend on important advances in our understanding of its risk factors. Self-rated health is a health measure based on the response to the simple question, “How do you feel?". Cumulative evidence suggests that self-rated health is a strong independent predictor for health outcomes such as morbidity and mortality [3],[4]. Further studies have shown that selfrated health status contains more important prognostic information than physician-assessed health status [5],[6]. Therefore, self-rated health has become an extensive measure of health in health research [7]-[9]. In addition, a report has shown that change in self-rated health is a stronger predictor of mortality than self-rated health at baseline and at the most recent observation [10]. Self-rated health is a changeable risk factor for death because self-rated health, as a subjective feeling of a person, reflects not only one person's physical and physiological health status, but also one person's psychosocial health status, such as quality of life, income and education [11]. Therefore, explorations of the determinants that have a significant effect on selfrated health and change in self-rated health status leading to establishing effective strategies for prolonging life in elder population are very important. 
The purpose of the present study is to assess the pattern of change in self-rated health, the determinants that affect self-rated health, and the change of self-rated health status and subsequent mortality prediction among community-dwelling elder Americans.

\section{MATERIALS AND METHODS}

The data used in this study were obtained from the 1994-2000 Second Longitudinal Studies of Aging (LSOA II), a publicly available database. The survey's design and procedures have been published elsewhere [12],[13]. In brief, the LSOA II is a collaborative effort between the National Center for Health Statistics (NCHS) and the National Institute on Aging. The goals of the LSOA II study are to better understand disability pathways and interrelationships between determinants and functional outcomes among elder adults. The LSOA II is a longitudinal study with a nationally representative sample consisting of 9,447 civilian non-institutionalized persons, 70 years of age and elder at the time of their baseline interview. This baseline interview, also known as the Second Supplement on Aging (SOA II), was conducted in the period of 1994-1996. The LSOA II followed this cohort of participants through two follow-up interviews during the periods of 1997-1998 and 1999-2000. The study sample has been linked with the National Death Index (NDI), called the LSOA II Linked Mortality File (CDC 2006). The LSOA II Linked Mortality File provides mortality follow-up data from the date of SOA II interview (1994-1996) through December 31, 2006. Mortality ascertainment is based primarily upon deaths previously identified during one of two follow-up interviews, in 1997-1998 and 1999-2000, and results from a probabilistic match between LSOA II and NDI death certificate records. Linkage of the LSOA II survey participants with the NDI provides the opportunity to conduct a vast array of outcome studies designed to investigate the association of a wide variety of health factors with mortality. In the present study, of the 9,447 subjects, there were 5,399 respondents who answered both self-rated health questions at baseline and 1999-2000 interviews. These respondents include 4,807 white Americans, 498 African Americans and 94 classified as “other”.

The measure of self-rated health in LSOA II was a single question asked at each interview: "Would you say your health in general is excellent, very good, good, fair, or poor?”. Responses to this question were coded 1 through 5, respectively. Chronic disease conditions of depression, cataracts, glaucoma, blindness, deafness, osteoporosis, diabetes, arthritis, bronchitis or emphysema, hypertension, asthma, heart disease, stroke and cancer are defined according to participants' self-reports of physician diagnosed disease at each interview ("whether a doctor told you that you had arthritis..."). The measure of activity of daily living difficulties was assessed by the number of items at each interview: "Because of a health or physical problem, do you have ANY difficulty?” The items included: bathing or showering, dressing, eating, getting in and out of bed or chairs, walking, using the toilet, preparing your own meals, shopping for groceries and personal items, managing your money, using the telephone, doing light housework, managing your medication. Social activities were determined using responses to the questions at each interview: "Did you get together with friends or neighbors?” and "Did you get together with relatives?”

For association analysis between self-rated health (or change in self-rated health) and demographic characteristic or risk factor, we constructed logistic regression models. These logistic regression models were constructed to calculate the odds ratios (OR) and confidence intervals (CI) of demographic characteristic and risk factors. All the odds ratios were adjusted with demographic factors, family factors, social factors, personal health behaviors factors, difficulties in daily activities and chronic diseases variables. For survival analysis, Cox proportional hazard regression model was constructed for examining the effects of self-rated health or change in self-rated health variables.

Analyses were conducted by using SAS software (version 9.2, SAS Institute, Cary, NC). A twosided p-value $<.05$ was considered to be statistically significant.

\section{RESULTS}

\subsection{Characteristics of Participants at baseline}

In table 1, the sample included 3,391 women and 2,008 men with an average age of 75.51 years ( $S D=5.25)$. The majority were White American $(n=4,807,89.04 \%)$. Of the 5,399 participants, 785(14.54\%) participants reported as self-rated excellent health status, 1,552(28.75\%) participants reported as self-rated very good health status, 1,974(36.56\%) participants reported as self-rated good health status, 863(15.98\%) participants reported as self-rated fair health status and 225(4.17\%) participants reported as self-rated poor health status. 
Table 1. Characteristics of participants in baseline survey, LSOAII between 1994 and 1996

\begin{tabular}{|c|c|c|c|}
\hline Characteristic & Mean (SD) or number (\%) & Characteristic & Mean (SD) or number (\%) \\
\hline Age & $75.51(5.25)$ & Number of chronic disease & $\begin{array}{l}0=853(15.80 \%) \\
1=1,276(23.63 \%)\end{array}$ \\
\hline Gender & $\begin{array}{l}\text { Male=2,008 }(37.19 \%) \\
\text { Female=3391 (62.81\%) }\end{array}$ & & $\begin{array}{l}2=1,265(23.43 \%) \\
3=941(17.43 \%) \\
4=552(10.22 \%)\end{array}$ \\
\hline BMI & 25.53 (4.39) & & $\begin{array}{l}5=305(5.65 \%) \\
6=133(2.46 \%)\end{array}$ \\
\hline Race & $\begin{array}{l}\text { White American=4,807 }(89.04 \%) \\
\text { African American=498 (9.22\%) } \\
\text { Other=94 (1.74\%) }\end{array}$ & & $\begin{array}{l}7=50(0.93 \%) \\
8=20(0.37 \%) \\
9=3(0.06 \%) \\
10=1(0.02 \%)\end{array}$ \\
\hline Education & $\begin{array}{l}\text { Elementary school=339 }(6.28 \%) \\
\text { Middle school=867 }(16.06 \%) \\
\text { High school=2,661 (49.29) } \\
\text { College=1,532 (28.38\%) }\end{array}$ & & \\
\hline Income & $\begin{array}{l}\text { Less than } \$ 20,000=2,877(53.29 \%) \\
\$ 20,000 \text { or more }=2,522(46.71 \%)\end{array}$ & $\begin{array}{l}\text { Number of difficulty in } \\
\text { daily activities }\end{array}$ & $\begin{array}{l}0=4,028(74.61 \%) \\
1=563(10.43 \%) \\
2=238(4.41 \%)\end{array}$ \\
\hline Marital status & $\begin{array}{l}\text { Married=2,853 }(52.84 \%) \\
\text { Widowed=2,018 }(37.38 \%) \\
\text { Divorced=276 (5.11\%) } \\
\text { Never married=252 (4.64\%) }\end{array}$ & & $\begin{array}{l}3=163(3.02 \%) \\
4=108(2.00 \%) \\
5=91(1.69 \%) \\
6=54(1.00 \%) \\
7=46(0.85 \%)\end{array}$ \\
\hline Family relationship & $\begin{array}{l}\text { Living alone }=1,823(33.77 \%) \\
\text { Living with spouse }=2,922(54.12 \%) \\
\text { Living with other }=654(12.11 \%)\end{array}$ & & $\begin{array}{l}8=40(0.74 \%) \\
9=26(0.48 \%) \\
10=15(0.28 \%) \\
11=14(0.26 \%)\end{array}$ \\
\hline $\begin{array}{l}\text { Together with friends } \\
\text { or neighbors }\end{array}$ & $\begin{array}{l}\text { Yes }=4,083(75.63 \%) \\
\mathrm{No}=1,316(24.37 \%)\end{array}$ & & $12=13(0.24 \%)$ \\
\hline $\begin{array}{l}\text { Together with } \\
\text { relatives }\end{array}$ & $\begin{array}{l}\text { Yes }=4,226(78.27 \%) \\
\text { No= } 1,173(21.73 \%)\end{array}$ & & \\
\hline Exercise & $\begin{array}{l}\text { Yes=2,328 }(43.12 \%) \\
\text { No=3,071 (56.88\%) }\end{array}$ & Self-rated health status & $\begin{array}{l}\text { Excellent }=785(14.54 \%) \\
\text { Very Good=1,552(28.75\%) } \\
\text { Good=1,974(36.56\%) }\end{array}$ \\
\hline Smoking & $\begin{array}{l}\text { Never smoked=2,946 }(54.57 \%) \\
\text { Current smoker=489 }(9.06 \%) \\
\text { Former smoker=1,936 (35.85\%) } \\
\text { Missing=28 (0.52\%) }\end{array}$ & & $\begin{array}{l}\text { Fair }=863(15.98 \%) \\
\text { Poor }=225(4.17 \%)\end{array}$ \\
\hline Alcohol consumption & $\begin{array}{l}\text { Yes=1,008 }(18.67 \%) \\
\text { No=4,391 (81.33\%) }\end{array}$ & & \\
\hline Region & $\begin{array}{l}\text { Northeast=1,155 (21.39\%) } \\
\text { Midwest=1,478 (27.38\%) } \\
\text { South=1,739 (32.21\%) } \\
\text { West=1,027 (19.02\%) } \\
\end{array}$ & & \\
\hline
\end{tabular}

\subsection{The effects of demographic characteristic or risk factor on self-rated health status}

Although self-rated health status is a subjective feeling, it can reflect the person's physiological or physical health status. As shown in table 2, people with more chronic disease conditions or more difficulty in daily activities were generally more likely to report "fair" or "poor" health condition. In order to combat these "spurious relationships", regression analysis was conducted. We constructed the logistic regression model for variable of self-rated health status (excellent, very good, good were coded as 1 , fair and poor were coded as 0 ). As shown in table 3 , compared to participants without chronic disease condition or difficulty in daily activities, participants with depress, osteoporosis, diabetes, arthritis, emphysema, hypertension, heart, cancer, difficulty of eating, difficulty of walking and difficulty of shopping are generally more likely to report "fair" or "poor" health status. In addition to chronic disease condition and difficulty in daily activities, other demographic characteristic and risk factors were also significantly correlated with self-rated health status. For example, compared with white American, African-Americans were less likely to report their selfrated health status as "excellent", "very good", or "good". Participants with less education or less income were more likely to report their self-rated health as "fair" or "poor". Exercise and getting together with 
friends or neighbors had positive effects on self-rated health status for participants (OR is 1.326 for exercise, OR is 1.584 for getting together with friends or neighbors).

Table 2. The effects of number of chronic disease or difficulty in daily activities on Self-rated health status

\begin{tabular}{|c|c|c|c|c|c|c|c|c|c|c|c|}
\hline \multicolumn{7}{|c|}{ Self-rated health status at baseline } & \multicolumn{5}{|c|}{ Self-rated health status at baseline } \\
\hline $\begin{array}{c}\text { No } \\
\text { Of chronic } \\
\text { disease }\end{array}$ & $\begin{array}{c}\text { Excellen } \\
\mathrm{t} \\
(\%)\end{array}$ & $\begin{array}{c}\text { Very } \\
\text { Good } \\
(\%)\end{array}$ & $\begin{array}{c}\text { Good } \\
(\%)\end{array}$ & $\begin{array}{l}\text { Fair } \\
(\%)\end{array}$ & $\begin{array}{c}\text { Poor } \\
(\%)\end{array}$ & $\begin{array}{c}\text { No } \\
\text { of } \\
\text { difficulty } \\
\text { in daily } \\
\text { activities }\end{array}$ & $\begin{array}{c}\text { Excellen } \\
\mathrm{t} \\
(\%)\end{array}$ & $\begin{array}{c}\text { Very } \\
\text { Good } \\
(\%)\end{array}$ & $\begin{array}{c}\text { Good } \\
(\%)\end{array}$ & $\begin{array}{l}\text { Fair } \\
\text { (\%) }\end{array}$ & $\begin{array}{c}\text { Poor } \\
(\%)\end{array}$ \\
\hline 0 & 28.25 & 34.70 & 30.48 & 5.74 & 0.82 & 0 & 17.68 & 33.42 & 36.99 & 10.90 & 1.02 \\
\hline 3 & 9.78 & 24.23 & 41.45 & 19.87 & 4.65 & 3 & 4.29 & 12.88 & 35.58 & 31.90 & 15.34 \\
\hline 4 & 5.80 & 18.66 & 40.94 & 26.45 & 8.15 & 4 & 7.41 & 8.33 & 22.22 & 40.74 & 21.30 \\
\hline 5 & 1.97 & 18.36 & 37.70 & 30.82 & 11.15 & 5 & 5.49 & 7.69 & 27.47 & 34.07 & 25.27 \\
\hline 6 & 2.26 & 9.77 & 32.33 & 37.59 & 18.05 & 6 & 1.85 & 14.81 & 18.52 & 35.19 & 29.63 \\
\hline 7 & 0.00 & 4.00 & 22.00 & 34.00 & 40.00 & 7 & 4.35 & 10.87 & 23.91 & 30.43 & 30.43 \\
\hline 11 & & & & & & 11 & 0.00 & 7.14 & 0.00 & 71.43 & 21.43 \\
\hline 12 & & & & & & 12 & 7.69 & 0.00 & 23.08 & 15.38 & 53.85 \\
\hline
\end{tabular}

Table 3. The association of self-rated health and demographic characteristic or risk factor

\begin{tabular}{|c|c|c|c|c|c|}
\hline $\begin{array}{l}\text { Demographic characteristic or } \\
\text { risk factor }\end{array}$ & OR & $(95 \%$ CI) & Demographic characteristic or risk factor & OR & (95\% CI) \\
\hline AGE & 1.009 & $\left(\begin{array}{ll}0.990 & 1.028\end{array}\right)$ & DEPRESSED ( Yes vs No) & 0.597 & $\left(\begin{array}{ll}0.441 & 0.809\end{array}\right)^{* *}$ \\
\hline GENDER (Female vs Male) & 1.007 & $(0.814$ 1.246) & CATARACTS (Yes vs No) & 1.074 & (0.883 1.308) \\
\hline RACE & & & GLAUCOMA (Yes vs No) & 0.798 & $(0.5891 .082)$ \\
\hline Black vs White & 0.539 & $(0.4080 .713)^{* * *}$ & & & \\
\hline Other vs White & 0.858 & $(0.4261 .729)$ & BLINDNESS (Yes vs No) & 0.815 & $\left(\begin{array}{lll}0.565 & 1.178\end{array}\right)$ \\
\hline BMI & 1.010 & $(0.9901 .031)$ & DEAFNESS (Yes vs No) & 0.902 & $(0.7061 .153)$ \\
\hline MARITAL_STATUS & & & OSTEOPOROSIS (Yes vs No) & 0.649 & $(0.4830 .872)^{* *}$ \\
\hline Divorced vs Married & 1.171 & $(0.6412 .141)$ & DIABETES (Yes vs No) & 0.656 & $(0.5070 .848)^{* *}$ \\
\hline Never married vs Married & 1.161 & $(0.6262 .152)$ & ARTHRITIS (Yes vs No) & 0.663 & $(0.5490 .799)^{* * *}$ \\
\hline Widowed vs Married & 1.174 & $\left(\begin{array}{ll}0.710 & 1.941\end{array}\right)$ & EMPHYSEMA (Yes vs No) & 0.547 & $(0.4130 .725)^{* * *}$ \\
\hline EDUCATION & & & ASTHMA (Yes vs No) & 0.764 & $(0.5351 .092)$ \\
\hline $\begin{array}{l}\text { Elementary school vs } \\
\text { college }\end{array}$ & 0.453 & $(0.3070 .669)^{* * *}$ & HYPERTENSION (Yes vs No) & 0.702 & $\left(\begin{array}{lll}0.587 & 0.840\end{array}\right)^{* *}$ \\
\hline High school vs college & 0.720 & $(0.5690 .913)^{* *}$ & HEART (Yes vs No) & 0.409 & $(0.3340 .501)^{* * *}$ \\
\hline Middle school vs college & 0.493 & $(0.3680 .659)^{* * *}$ & STROKE (Yes vs No) & 0.730 & $(0.5331 .000)$ \\
\hline INCOME & & & CANCER (Yes vs No) & 0.793 & $(0.6310 .995)^{*}$ \\
\hline $\begin{array}{l}\text { Less than } \$ 20,000 \text { vs } \\
\$ 20,000 \text { or more }\end{array}$ & 0.665 & $\left(\begin{array}{lll}0.541 & 0.817\end{array}\right)^{* * *}$ & & & \\
\hline RELATIONSHIP & & & DIFFICULTY_BATHING (Yes vs No) & 0.732 & $\left(\begin{array}{ll}0.510 & 1.050\end{array}\right)$ \\
\hline $\begin{array}{l}\text { Living alone vs Living with } \\
\text { spouse }\end{array}$ & 1.068 & $(0.643$ 1.776) & DIFFICULTY_DRESSING (Yes vs No) & 0.733 & $(0.471$ 1.142) \\
\hline $\begin{array}{l}\text { Living with other vs Living } \\
\text { with spouse }\end{array}$ & 0.863 & $\left(\begin{array}{lll}0.506 & 1.471\end{array}\right)$ & DIFFICULTY_EATING (Yes vs No) & 0.468 & $\left(\begin{array}{lll}0.230 & 0.953\end{array}\right)^{*}$ \\
\hline FRIENDS (Yes vs No) & 1.584 & $(1.3041 .923)^{* * *}$ & DIFFICULTY_BED1 (Yes vs No) & 0.725 & $(0.5141 .024)$ \\
\hline RELATIVES (Yes vs No) & 0.952 & $(0.7691 .178)$ & DIFFICULTY_WALKING (Yes vs No) & 0.464 & $(0.3620 .595)^{* * *}$ \\
\hline EXERCISE (Yes vs No) & 1.326 & $(1.1041 .592)^{* *}$ & DIFFICULTY_TOILET1 (Yes vs No) & 1.627 & (0.956 2.769) \\
\hline SMOKE (Yes vs No) & 0.751 & $(0.5631 .003)$ & DIFFICULTY_MEALS1 (Yes vs No) & 0.830 & $(0.5141 .339)$ \\
\hline ALCOHOL (Yes vs No) & 1.242 & $(0.9621 .604)$ & DIFFICULTY_SHOPPING (Yes vs No) & 0.456 & $(0.3240 .640)^{* * *}$ \\
\hline REGION & & & DIFFICULTY_MONEY1 (Yes vs No) & 1.194 & $(0.6662 .142)$ \\
\hline Midwest vs Northeast & 1.019 & $\left(\begin{array}{ll}0.790 & 1.316\end{array}\right)$ & DIFFICULTY_TELEPHONE(Yesvs No) & 0.667 & $(0.3891 .144)$ \\
\hline South vs Northeast & 0.838 & $(0.654$ 1.072) & $\begin{array}{l}\text { DIFFICULTY_HOUSEWORK (Yes vs } \\
\text { No) }\end{array}$ & 0.757 & (0.483 1.188) \\
\hline West vs Northeast & 0.971 & $\left(\begin{array}{ll}0.727 & 1.297\end{array}\right)$ & DIFFICULTY_MEDICATIO (Yes vs No) & 0.968 & $\left(\begin{array}{lll}0.515 & 1.822\end{array}\right)$ \\
\hline
\end{tabular}




\subsection{The pattern of changed responses to self-rated health question}

Self-rated health status would change after an individual's physical, physiological, or psychosocial health status changed over time. As shown in tables 4 and 5, a substantial proportion of participants (59.07\%) changed their reported health status after a 6-year follow-up, 39.17\% downgraded their self-rated health status and $19.88 \%$ upgraded their self-rated health status. Among participants who rated their health as "excellent" at baseline, $70.45 \%$ changed their rating; make it the least stable of the 5 self-rated health levels. Only 51.56\% participants who reported their health as "poor" at baseline change their rating; making it the most stable level of 5 self-rated health categories. In addition, $42.91 \%$ participants changed ratings by only one level, while $16.16 \%$ of the participants changed their ratings by more than one level.

Table 4. The pattern of changed responses to self-rated health question by categories

\begin{tabular}{|c|c|c|c|c|c|c|}
\hline \multirow{2}{*}{\multicolumn{2}{|c|}{$\begin{array}{c}\text { Self-rated health } \\
\text { Interview (1994-1996) }\end{array}$}} & \multicolumn{5}{|c|}{$\begin{array}{c}\text { Self-rated health } \\
\text { Interview (1999-2000) }\end{array}$} \\
\hline & & \multirow{2}{*}{$\begin{array}{c}\text { Excellent } \\
4.30 \%(232)\end{array}$} & \multirow{2}{*}{$\begin{array}{c}\text { Very Good } \\
5.87 \%(317)\end{array}$} & \multirow{2}{*}{$\begin{array}{c}\text { Good } \\
3.09 \%(167)\end{array}$} & \multirow{2}{*}{$\begin{array}{c}\text { Fair } \\
0.80 \%(43)\end{array}$} & \multirow{2}{*}{$\begin{array}{c}\text { Poor } \\
0.48 \%(26)\end{array}$} \\
\hline Excellent & $14.54 \%(785)$ & & & & & \\
\hline Very Good & $28.75 \%(1552)$ & $2.89 \%(156)$ & $10.96 \%(592)$ & $10.09 \%(545)$ & 3.45\%(186) & $1.35 \%(73)$ \\
\hline Good & $36.56 \%(1974)$ & $1.57 \%(85)$ & 7.35\%(397) & $16.80 \%(907)$ & $8.00 \%(432)$ & $2.83 \%(153)$ \\
\hline Fair & $15.98 \%(863)$ & $0.39 \%(21)$ & $1.33 \%(72)$ & $4.20 \%(227)$ & $6.85 \%(370)$ & $3.20 \%(173)$ \\
\hline Poor & $4.17 \%(225)$ & $0.07 \%(4)$ & $0.20 \%(11)$ & $0.57 \%(31)$ & $1.30 \%(70)$ & $2.02 \%(109)$ \\
\hline Total & $100.00 \%(5399)$ & $9.22 \%(498)$ & $25.73 \%(1389)$ & $34.77 \%(1877)$ & $20.39 \%(1101)$ & $9.89 \%(534)$ \\
\hline
\end{tabular}

Table 5.The pattern of changed responses to self-rated health question by levels

\begin{tabular}{|c|c|c|c|c|c|c|c|c|c|c|}
\hline & \multirow[t]{2}{*}{$\begin{array}{c}\text { Interview } \\
(1994- \\
1996) \\
\end{array}$} & \multicolumn{9}{|c|}{$\begin{array}{l}\text { Changed level of self-rated health } \\
\text { Interview (1999-2000) }\end{array}$} \\
\hline & & -4 & -3 & -2 & -1 & 0 & 1 & 2 & 3 & 4 \\
\hline Excellent & $\begin{array}{c}14.54 \% \\
(785)\end{array}$ & $\begin{array}{c}0.48 \% \\
(26)\end{array}$ & $\begin{array}{c}0.80 \% \\
(43)\end{array}$ & $\begin{array}{c}3.09 \% \\
(167)\end{array}$ & $\begin{array}{c}5.87 \% \\
(317)\end{array}$ & $\begin{array}{c}4.30 \% \\
(232)\end{array}$ & $\begin{array}{c}0.00 \% \\
(0)\end{array}$ & $\begin{array}{c}0.00 \% \\
(0)\end{array}$ & $\begin{array}{c}0.00 \% \\
(0)\end{array}$ & $\begin{array}{c}0.00 \% \\
(0)\end{array}$ \\
\hline Very Good & $\begin{array}{c}28.75 \% \\
(1552)\end{array}$ & $\begin{array}{l}0.00 \% \\
(0)\end{array}$ & $\begin{array}{l}1.35 \% \\
(73)\end{array}$ & $\begin{array}{l}3.45 \% \\
(186)\end{array}$ & $\begin{array}{c}10.09 \% \\
(545)\end{array}$ & $\begin{array}{c}10.96 \% \\
(592)\end{array}$ & $\begin{array}{l}2.89 \% \\
(156)\end{array}$ & $\begin{array}{l}0.00 \% \\
(0)\end{array}$ & $\begin{array}{l}0.00 \% \\
(0)\end{array}$ & $\begin{array}{c}0.00 \% \\
(0)\end{array}$ \\
\hline Good & $\begin{array}{c}36.56 \% \\
(1974)\end{array}$ & $\begin{array}{c}0.00 \% \\
(0)\end{array}$ & $\begin{array}{c}0.00 \% \\
(0)\end{array}$ & $\begin{array}{c}2.83 \% \\
(153)\end{array}$ & $\begin{array}{c}8.00 \% \\
(432)\end{array}$ & $\begin{array}{c}16.80 \% \\
(907)\end{array}$ & $\begin{array}{c}7.35 \% \\
(397)\end{array}$ & $\begin{array}{c}1.57 \% \\
(85)\end{array}$ & $\begin{array}{c}0.00 \% \\
(0)\end{array}$ & $\begin{array}{c}0.00 \% \\
(0)\end{array}$ \\
\hline Fair & $\begin{array}{c}15.98 \% \\
(863)\end{array}$ & $\begin{array}{c}0.00 \% \\
(0)\end{array}$ & $\begin{array}{c}0.00 \% \\
(0)\end{array}$ & $\begin{array}{c}0.00 \% \\
(0)\end{array}$ & $\begin{array}{c}3.20 \% \\
(173)\end{array}$ & $\begin{array}{c}6.85 \% \\
(370)\end{array}$ & $\begin{array}{c}4.20 \% \\
(227)\end{array}$ & $\begin{array}{c}1.33 \% \\
(72)\end{array}$ & $\begin{array}{c}0.39 \% \\
(21)\end{array}$ & $\begin{array}{c}0.00 \% \\
(0)\end{array}$ \\
\hline Poor & $\begin{array}{l}4.17 \% \\
(225)\end{array}$ & $\begin{array}{c}0.00 \% \\
(0)\end{array}$ & $\begin{array}{c}0.00 \% \\
(0)\end{array}$ & $\begin{array}{l}0.00 \% \\
(0)\end{array}$ & $\begin{array}{c}0.00 \% \\
(0)\end{array}$ & $\begin{array}{l}2.02 \% \\
(109)\end{array}$ & $\begin{array}{c}1.30 \% \\
(70)\end{array}$ & $\begin{array}{c}0.57 \% \\
(31) \\
\end{array}$ & $\begin{array}{c}0.20 \% \\
(11) \\
\end{array}$ & $\begin{array}{c}0.07 \% \\
(4)\end{array}$ \\
\hline Total & $\begin{array}{c}100.00 \% \\
(5399)\end{array}$ & $\begin{array}{c}0.48 \% \\
(26)\end{array}$ & $\begin{array}{c}2.15 \% \\
(116)\end{array}$ & $\begin{array}{l}9.37 \% \\
(506)\end{array}$ & $\begin{array}{c}27.17 \% \\
(1467)\end{array}$ & $\begin{array}{l}40.93 \% \\
(2210)\end{array}$ & $\begin{array}{c}15.74 \% \\
(850)\end{array}$ & $\begin{array}{c}3.48 \% \\
(188)\end{array}$ & $\begin{array}{c}0.59 \% \\
(32)\end{array}$ & $\begin{array}{c}0.07 \% \\
(4)\end{array}$ \\
\hline
\end{tabular}

Percentage (number)

\subsection{Determinants that affect the change of self-rated health status}

Although many studies have found that self-rated health is associated with demographic characteristics or risk factors, no study has explored the determinants that affect the change in self-rated health status. Therefore, we are exploring which factors, such as demographic characteristic, change of physical, physiological or psychosocial health status, have a significant effect on change in self-rated health status. In table 6, we constructed the logistic regression model for variables of change in self-rated health status (downgrade or upgrade versus no change). Several stable variables, such as gender, race, education, region and income, and changed variables, such as age, changed BMI, changed exercise, changed friends, changed relatives, changed difficulty in daily activities and changed chronic disease conditions, were included in this model. As shown in table 6, age, gender, race, education, region and income were not significantly associated with change in self-rated health status. Just as participants with difficulty in daily activities or chronic disease conditions were more likely report to be "fair" or "poor" health status, participants with change from "no difficulty" to "difficulty" in daily activities or change from "normal" to "diseases" were more likely to change their self-rated health status to downgrade. For example, participants were likely to downgrade their self-rated health for changed difficulty of walking, cooking meals, heart disease and stroke. Continuous difficulty of dressing and toilet was also more likely to downgrade their selfrated health status. Very interestingly, participants with release from difficulty in daily activities, such as 
eating and bed, were more likely to upgrade their status. Participants with release from suspending or misdiagnosis with diabetes or emphysema were also more likely to upgrade their status.

Table 6. The association between change in self-rated health and demographic characteristic

\begin{tabular}{|c|c|c|c|c|c|c|c|c|c|}
\hline \multirow{3}{*}{ Age } & $O R$ & $95 \% C I$ & OR & $95 \% C I$ & & $O R$ & $95 \% C I$ & $O R$ & $95 \% C I$ \\
\hline & \multicolumn{2}{|c|}{ Downgrade } & \multicolumn{2}{|c|}{ Upgrade } & & \multicolumn{2}{|c|}{ Downgrade } & \multicolumn{2}{|c|}{ Upgrade } \\
\hline & 0.997 & $(0.977$ & 1.021 & (0.997 & \multirow{2}{*}{\multicolumn{3}{|c|}{ Change difficulty telephone }} & & \\
\hline & & 1.017) & & $1.044)$ & & & & & \\
\hline \multirow[t]{2}{*}{ Gender ( $\mathrm{F}$ vs M) } & 0.869 & $(0.714$ & 0.920 & $(0.730$ & 2 vs 1 & 1.172 & $(0.785$ & 1.511 & $(0.941$ \\
\hline & & 1.056) & & $1.160)$ & & & $1.750)$ & & 2.426) \\
\hline \multirow[t]{2}{*}{ Race } & & & & & 3 vs 1 & 0.592 & $(0.139$ & 1.056 & $(0.271$ \\
\hline & & & & & & & 2.528) & & $4.122)$ \\
\hline \multirow[t]{2}{*}{ Black vs White } & 1.170 & $(0.852$ & 1.127 & $(0.778$ & 4 vs 1 & 0.424 & $(0.146$ & 0.900 & $(0.278$ \\
\hline & & 1.607) & & 1.634) & & & 1.231) & & 2.913) \\
\hline \multirow[t]{2}{*}{ Others vs White } & 0.905 & $(0.431$ & 1.248 & $(0.553$ & \multicolumn{3}{|c|}{ Change difficulty housework } & & \\
\hline & & $1.904)$ & & 2.815) & & & & & \\
\hline Education & & & & & 2 vs 1 & 0.674 & $(0.408$ & 1.162 & $(0.616$ \\
\hline & & & & & & & 1.114) & & 2.189) \\
\hline Elementary vs & 1.319 & $(0.838$ & 1.300 & $(0.763$ & 3 vs 1 & 0.991 & $(0.423$ & 1.457 & $(0.643$ \\
\hline college & & 2.075) & & 2.213) & & & 2.321) & & 3.300) \\
\hline Middle vs college & 0.962 & $(0.710$ & 1.054 & $(0.746$ & 4 vs 1 & 0.751 & $(0.281$ & 0.853 & $(0.278$ \\
\hline & & $1.302)$ & & $1.490)$ & & & 2.011) & & 2.615) \\
\hline High vs college & 0.992 & $(0.814$ & 0.956 & $(0.757$ & Chang & lity med & & & \\
\hline & & 1.209) & & 1.208) & & & & & \\
\hline Region & & & & & 2 vs 1 & 1.594 & $(0.940$ & 1.324 & $(0.691$ \\
\hline & & & & & & & 2.703) & & 2.538) \\
\hline Midwest vs & 1.143 & $(0.897$ & 1.170 & $(0.883$ & 3 vs 1 & 0.542 & $(0.100$ & 0.199 & $(0.031$ \\
\hline Northeast & & $1.457)$ & & $1.551)$ & & & 2.946) & & $1.274)$ \\
\hline South vs Northeast & 0.998 & $(0.785$ & 1.070 & $(0.810$ & 4 vs 1 & 1.496 & $(0.466$ & 0.374 & $(0.083$ \\
\hline & & 1.268) & & 1.413) & & & $4.798)$ & & $1.687)$ \\
\hline West vs Northeast & 1.117 & 0.8631 .446 & 0.891 & $(0.652$ & Chang & & & & \\
\hline & & & & 1.217) & & & & & \\
\hline Income & & & & & 2 vs 1 & 0.951 & $(0.763$ & 0.873 & $(0.668$ \\
\hline (Less than $\$ 20,00$ vs & 1.121 & $(0.935$ & 1.063 & $(0.858$ & & & 1.187) & & $1.139)$ \\
\hline$\$ 20,000$ or more ) & & $1.344)$ & & 1.318) & & & & & \\
\hline Change BMI & & & & & 3 vs 1 & 1.010 & $(0.768$ & 1.312 & $(0.976$ \\
\hline & & & & & & & 1.329) & & $1.762)$ \\
\hline Decrease vs no & 1.021 & $(0.789$ & 0.954 & $(0.705$ & 4 vs 1 & 1.172 & $(0.905$ & 0.813 & $(0.589$ \\
\hline change & & $1.323)$ & & $1.292)$ & & & 1.519) & & $1.121)$ \\
\hline Increase vs no & 0.943 & $(0.714$ & 1.016 & $(0.736$ & Chang & ma & & & \\
\hline change & & $1.245)$ & & $1.402)$ & & & & & \\
\hline Change exercise & & & & & 2 vs 1 & 1.418 & $(0.961$ & 1.148 & $(0.717$ \\
\hline & & & & & & & 2.093) & & 1.838) \\
\hline 2 vs 1 & 0.835 & $(0.649$ & 0.861 & $(0.644$ & 3 vs 1 & 1.602 & $(0.814$ & 1.273 & $(0.569$ \\
\hline & & 1.073) & & 1.151) & & & $3.154)$ & & $2.846)$ \\
\hline 3 vs 1 & 1.147 & $(0.890$ & 0.764 & $(0.553$ & 4 vs 1 & 0.905 & $(0.620$ & 0.943 & $(0.608$ \\
\hline & & $1.477)$ & & $1.054)$ & & & $1.322)$ & & $1.464)$ \\
\hline 4 vs 1 & 0.905 & $(0.734$ & 0.946 & $(0.744$ & Chang & & & & \\
\hline & & 1.116) & & $1.204)$ & & & & & \\
\hline Change friends & & & & & 2 vs 1 & 1.042 & $(0.706$ & 0.973 & $(0.603$ \\
\hline & & & & & & & $1.537)$ & & $1.571)$ \\
\hline 2 vs 1 & 1.084 & $(0.740$ & 0.827 & $(0.538$ & 3 vs 1 & 0.451 & $(0.159$ & 0.675 & $(0.247$ \\
\hline & & $1.588)$ & & $1.271)$ & & & $1.277)$ & & $1.846)$ \\
\hline 3 vs 1 & 1.169 & $(0.831$ & 0.849 & $(0.577$ & 4 vs 1 & 0.904 & $(0.536$ & 1.212 & $(0.673$ \\
\hline & & $1.644)$ & & $1.250)$ & & & 1.523) & & $2.182)$ \\
\hline 4 vs 1 & 1.187 & $(0.871$ & 0.956 & $(0.681$ & Chang & & & & \\
\hline & & 1.620) & & $1.343)$ & & & & & \\
\hline Change relatives & & & & & 2 vs 1 & 1.064 & $(0.833$ & 1.042 & $(0.780$ \\
\hline & & & & & & & $1.359)$ & & $1.391)$ \\
\hline 2 vs 1 & 0.896 & $(0.620$ & 0.835 & $(0.545$ & 3 vs 1 & 0.914 & $(0.564$ & 0.886 & $(0.507$ \\
\hline & & $1.295)$ & & $1.280)$ & & & $1.483)$ & & $1.546)$ \\
\hline 3 vs 1 & 0.865 & $(0.614$ & 0.845 & $(0.572$ & 4 vs 1 & 0.983 & $(0.717$ & 0.883 & $(0.609$ \\
\hline & & 1.218) & & $1.248)$ & & & 1.348) & & $1.281)$ \\
\hline 4 vs 1 & 0.875 & $(0.655$ & 0.877 & $(0.632$ & Chang & orosis & & & \\
\hline & & $1.170)$ & & $1.217)$ & & & & & \\
\hline Change difficulty batl & & & & & 2 vs 1 & 1.130 & $(0.874$ & 1.083 & $(0.794$ \\
\hline & & & & & & & $1.461)$ & & $1.476)$ \\
\hline 2 vs 1 & 0.866 & $(0.598$ & 1.271 & $(0.818$ & 3 vs 1 & 0.956 & $(0.500$ & 1.419 & $(0.728$ \\
\hline & & $1.254)$ & & 1.975) & & & 1.829) & & $2.766)$ \\
\hline 3 vs 1 & 1.231 & $(0.552$ & 1.663 & $(0.752$ & 4 vs 1 & 1.178 & $(0.814$ & 1.206 & $(0.791$ \\
\hline & & 2.744) & & 3.678) & & & 1.707) & & 1.839) \\
\hline
\end{tabular}




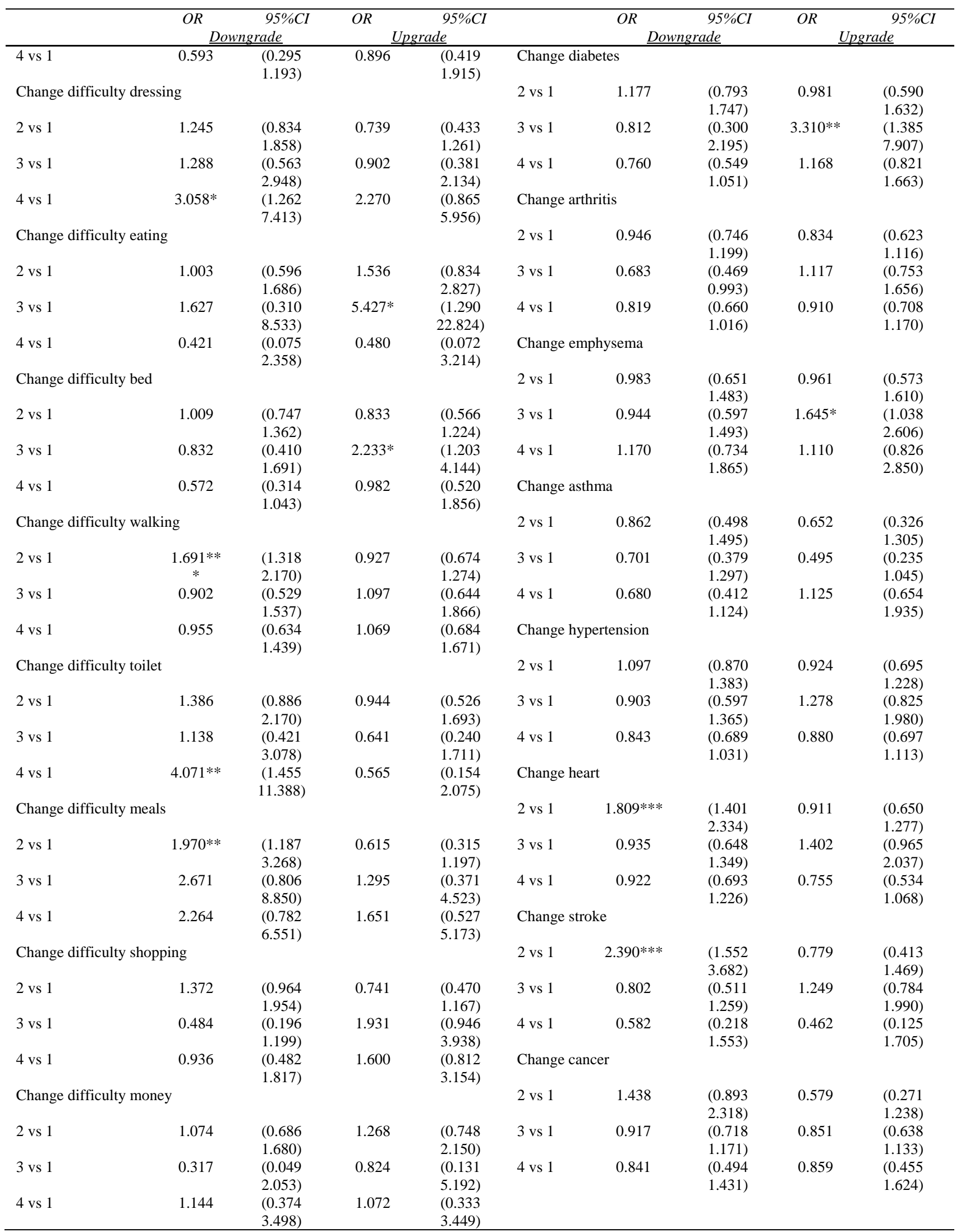

Odds ratios (OR) for (downgrade or upgrade) changed self-rated health and their 95\% confidence intervals (CI), model adjusted for other demographic, disease conditions and other risk factors. ${ }^{* * *} \mathrm{p}<0.0001,{ }^{* *} \mathrm{p}<0.01,{ }^{*} \mathrm{p}<0.05 .1$ represents "No" at baseline and "No" at 1999-2000; 2 represents "No" at baseline and "Yes" at 1999-2000; 3 represents "Yes" at baseline and "No" at 1999-2000; 4 represents "Yes" at baseline and "Yes" at 1999-2000. 


\subsection{Change in self-rated health status and survival analysis}

Of the 5,399 participants within a twelve-year follow-up, 2,647(49.03\%) were deceased by December 31, 2006. We did survival function estimation to compare analysis of participants with different values of change in self-rated health. As shown in figure 1A, there was higher mortality in downgrade and upgrade groups than group without self-rated health change. When the changed levels were taken into account, the higher the level changed, the worse the survival function $(\mathrm{p}<0.0001)$ for all changed levels versus no change (figure 1B). As shown in table 7, Cox proportional hazard regression model also showed the same results.

Self-rated health has been shown a strong predictor of mortality. Change in self-rated health is also showed to be a predictor of survival function. Then, this raises the question, what are the results when selfrated health is assessed for mortality when taking change in self-rated health status into account? To examine this, we constructed and evaluated the Cox models between self-rated health and subsequence health outcomes when incorporating changed self-rated health experience with the most recent self-rated health condition. Table 8 showed the results of Cox proportional hazard regression models 1-4 on the association between self-rated health and mortality at the bivariate level. Model 1 represents self-rated health status at baseline. Model 2 represents self-rated health status in an interview during 1999-2000. The value of change in self-rated health was considered in Model 3 and 4. Compared with self-rated health at baseline, self-rated health at the interview of 1999-2000 was a stronger predictor of mortality. Furthermore, when change in selfrated health status was considered, self-rated health was a stronger and more flexible predictor of mortality. With each additional 1 unit of decline in self-rated health, participants with a downgraded change of selfrated health were more likely to die than participants with an upgraded change of self-rated health(HR=1.647 vs 1.355). Models 3 and 4 take current self-rated health status and history self-rated health change into account for mortality prediction.
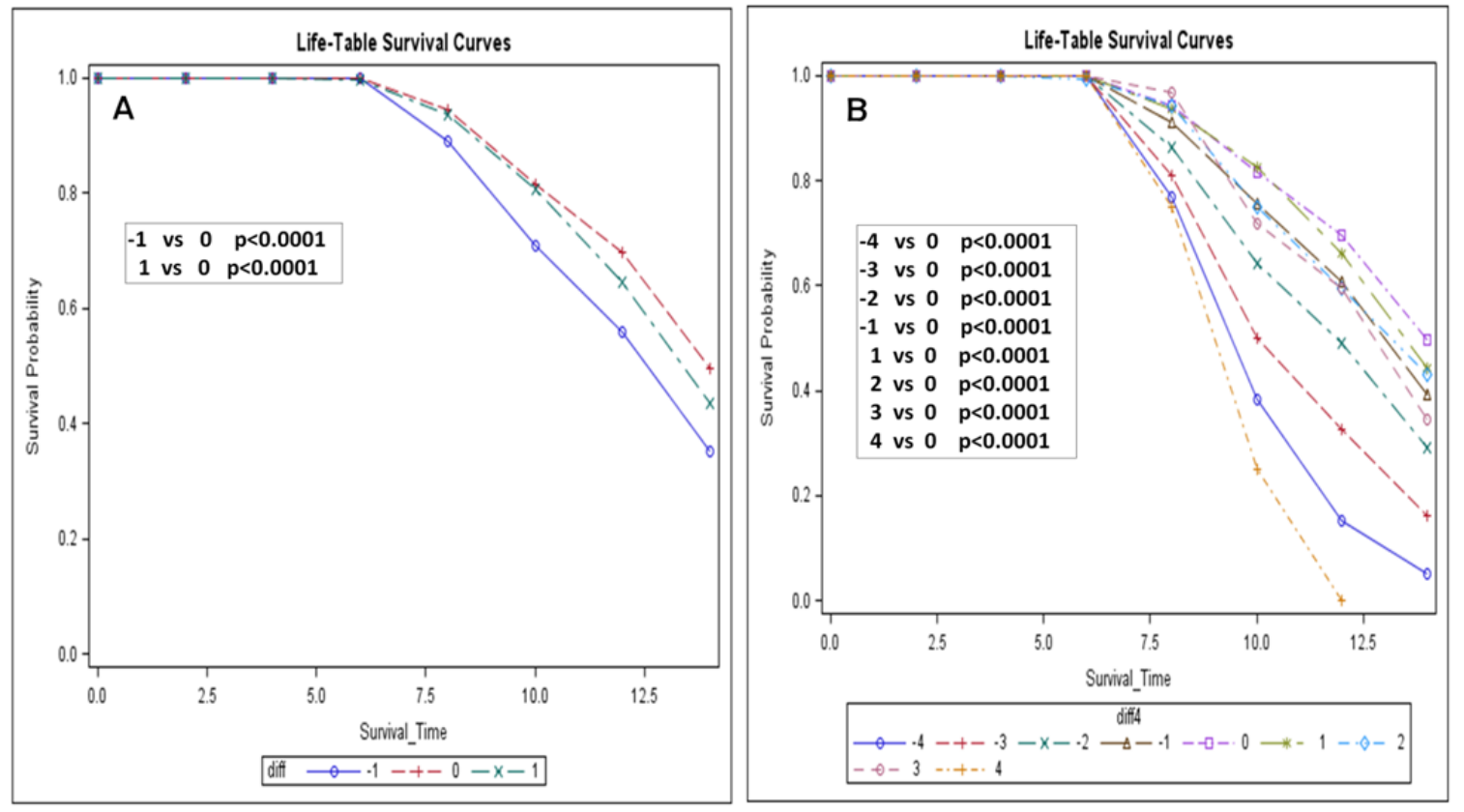

Figure 1. The effects of change of self-rated health on survival function

There was higher mortality in downgrade and upgrade groups than group without self-rated health change with $\mathrm{P}<0.0001$ (diff -1 : downgrade; 0: no change; 1: upgrade in Figure 1.A). When the levels were taken into account, the higher the level changed, the worse the survival function $(\mathrm{p}<0.0001)$ for all changed levels versus no change (diff4 -4: downgrade 4 levels; -3: downgrade 3 levels; -2: downgrade 2 levels; -1 : downgrade 1 level; 0: no change; 1: upgrade 1 level; 2: upgrade 2 levels; 3: upgrade 3 levels; 4: upgrade 4 levels in Figure 1.B). 
Table 7. The effects of change of self-rated health on hazard ratios

\begin{tabular}{|c|c|c|c|}
\hline & Hazard Ratio & 95\% Hazard RatioCI & $P$-value \\
\hline Upgrade vs no change & 1.186 & 1.065 & 0.0019 \\
\hline-4 vs 0 & 5.010 & 7.518 & $<.0001$ \\
\hline-3 vs 0 & 3.060 & 3.796 & $<.0001$ \\
\hline-2 vs 0 & 1.938 & 2.201 & $<.0001$ \\
\hline 2 vs 0 & 1.299 & 1.606 & 0.0158 \\
\hline 3 vs 0 & 1.500 & 0.941 & 0.0886 \\
\hline 4 vs 0 & 6.847 & $2.563 \quad 18.287$ & 0.0001 \\
\hline
\end{tabular}

Changed level of self-rated health in 9 levels: -4: downgrade 4 levels; -3: downgrade 3 levels; -2: downgrade 2 levels;-1: downgrade 1 level; 0: no change; 1: upgrade 1 level; 2: upgrade 2 levels; 3: upgrade 3 levels; 4: upgrade 4 levels.

Table 8. The effects of self-rated health with change of self-rated health level on hazard ratios

\begin{tabular}{|c|c|c|c|c|c|c|}
\hline Model & variable & $\begin{array}{l}\text { Changed level of } \\
\text { self-rated health }\end{array}$ & Hazard Ratio & \multicolumn{2}{|c|}{ 95\% Hazard Ratio CI } & P-value \\
\hline 1 & Baseline self-rated health & & 1.282 & \multicolumn{2}{|c|}{$(1.2361 .331)$} & $<.0001$ \\
\hline 2 & The most recent self-rated health & & 1.513 & \multicolumn{2}{|c|}{ (1.459 1.568) } & $<.0001$ \\
\hline \multirow[t]{4}{*}{3} & \multirow[t]{4}{*}{ The most recent self-rated health } & 0 & 1.563 & \multicolumn{2}{|c|}{ (1.462 1.670) } & $<.0001$ \\
\hline & & 1 & 1.355 & \multirow{2}{*}{\multicolumn{2}{|c|}{ (1.225 1.499) }} & $<.0001$ \\
\hline & & -4 & NA & & & \\
\hline & & -1 & 1.553 & 1.440 & 1.676 & $<.0001$ \\
\hline \multirow[t]{4}{*}{4} & \multirow[t]{4}{*}{ The most recent self-rated health } & 0 & 1.563 & 1.462 & 1.670 & $<.0001$ \\
\hline & & 1 & 1.441 & 1.283 & 1.619 & $<.0001$ \\
\hline & & 2 & 1.520 & 1.160 & 1.992 & 0.0024 \\
\hline & & 3 & 1.356 & 0.525 & 3.505 & 0.5294 \\
\hline
\end{tabular}

Changed level of self-rated health in model 3: -1: downgrade; 0: no change; 1: upgrade. Changed level of self-rated health in model 4: -4: downgrade 4 levels; -3: downgrade 3 levels; -2: downgrade 2 levels; -1: downgrade 1 level; 0: no change; 1: upgrade 1 level; 2: upgrade 2 levels; 3: upgrade 3 levels; 4: upgrade 4 levels.

\section{DISCUSSION}

In this study, we examined the changed pattern of self-rated health among community-dwelling elder Americans during a period between 1994 and 2000. Furthermore, we systematically assessed the associated factors that affect self-rated health and the change of self-rated health status and subsequent mortality prediction with self-rated health and change in self-rated health.

As an important risk factor for death, self-rated health is determined by several factors. In general, an individual's medical health status and/or physical function are major factors that contribute to self-rating health [14]. Our results provide further evidence that people with more chronic disease conditions or more difficulty in daily activities were generally more likely to report "fair" or "poor" health condition than the people with less chronic diseases or less difficulty in daily activities. Furthermore, medical health status, such as depression, osteoporosis, diabetes, arthritis, emphysema, hypertension, heart disease, cancer, and physical function, such as difficulty eating, walking or shopping, are significantly associated with self-rated health. In addition to chronic disease condition and difficulty in daily activities, some demographic characteristic, such as race, education and income are significantly correlated with self-rated health status [15]. Furthermore, health behaviors, such as exercise, and social activity are also associated with self-related health.

Because it reflects an individual's current medical, physical and psychosocial health status, selfrated health is likely to change over time. Our result showed that $59 \%$ of participants changed their reported health status after a 6-year follow-up, 39\% downgraded their self-rated health status and $20 \%$ upgraded their self-rated health status. About $40 \%$ of young people changed their self-rated health over a 4-year period [16],[17]. For a short-term period, one study reported that only about $15 \%$ of respondents changed their selfrated health after a one month follow up [18]. Recently, another study showed that about $40 \%$ of respondents changed their health rating about 1 month later due to different interview settings [11]. Therefore, although individuals' self-rated health status will change over time, the change in self-rated health will vary depending on population and period of following time. For example, a substantial proportion of participants (from 15\% to $40 \%$ ) changed their reported health status between the different interviews and response reliability was related to socio-demographic factors, such as age, income and education [19]-[21]. As for elder population, 
although participants can change in both directions, more participants will change their self-rated health from higher level to lower level.

Just as self-rated health, change in self-rated health is also a risk factor for mortality. Therefore, exploration of the determinants of change in self-rated health status is very important. Although changes in self-rated health for short-term periods reflect more unreliability [11],[18],[19], changes in self-rated health for long-term periods reflect more health status change [22],[23]. Our results show that, not like self-rated health, some demographic characteristic, such as age, gender, race, education and income, are not significantly associated with changes of self-rated health for community-dwelling elder Americans. In agreement with the findings from Finnish [22],[23], difficulties in daily activities are significantly associated with self-rated health for elder Americans. For example, participants were likely to downgrade their selfrated health for changed difficulty of walking, cooking meals, heart disease and stroke. Very interestingly, participants with release from difficulty in daily activities, such as eating and bed, were more likely to upgrade their status. Continuous difficulty of dressing and toilet also was more likely to downgrade their selfrated health status when compared with participants without changed difficulty in daily activities. Just as with difficulty in daily activities, participants were more likely to downgrade their self-rated health status when their medical health status changed from "normal" to "diseases", such as heart disease and stroke. In addition, participants with release from suspending or misdiagnosis with diabetes or emphysema were also more likely to upgrade their status. Together, chronic disease conditions and difficulties in daily activities were the main reasons for change in self-rated health status. Although the mechanism is not well understood, both pain and particular functional difficulties associated with these chronic disease conditions may be involved in the association between self-rated health status and chronic diseases [24],[25]. Therefore, for these elderly people with pain or functional difficulty, necessary syndrome treatment, and physical support are essential for improving their self-rated health status. In addition, if an individual downgrades her/his selfrated health with no apparent reason, her/his physiological, physical or psychological health condition need to be re-estimated.

Although many studies have explored the relationship between self-rated health and survival function, few have explored the relationship between changed self-rated health status and survival function or the relationship between self-rated health and survival function when taking account into changed self-rated health condition. Our results clearly showed that change in self-rated health have a significant impact on survival function in the elderly population. No matter whether participants upgrade or downgrade their selfrated health, participants with change in self-rated health status had a significantly decreased survival function when compared with people without change in self-rated health status during a 6-year follow-up. According to Cox proportional hazard regression models, each additional 1 unit of decline in baseline selfrated health, participants were 1.282 times more likely to die. With each additional 1 unit of decline in the most recent self-rated health, participants were 1.513 times more likely to die. Consistent with the study by Han et al [26], our study provides further evidence that although what participants report their current selfrated health to be is important, how they arrive at their current self-rated health from previous self-rated health is also very important. For example, when change in self-rated health status was considered, the results were stronger and more flexible. With each additional 1 unit of decline in most recent self-rated health, participants with a downgraded change of self-rated health were more likely to die than participants with an upgraded change of self-rated health. Therefore, the most recent self-rated health is a more predictive value when cooperating with change in self-rated health.

Several limitations in this study should be taken into account. First, the racial makeup of the population is not evenly distributed, 93\% population is white Americans, and only 7\% population is African Americans. Second, because the LSOA II study does not include laboratory data, we were unable to examine whether any biomarkers could be the potential mediators of the associations between self-rated health and death in American population. Finally, because the data for this study came from community-dwelling participants, individuals with severe disease status, such as hospitalized patients, may not have been included in this survey dataset. Strengths of this study include the use of a prospective cohort design that involves a representative sample of elderly people living in the community. Responses rates are high and complete mortality evaluations were found using the National Death Index system which minimizes potential death certificate reporting bias.

Despite some limitations, the results from this study provide further evidence that chronic disease conditions and difficulties in daily activities are the main reasons for change in self-rated health status in the elderly American population. Therefore, for these elderly people with chronic disease conditions and difficulties in daily activities, necessary syndrome treatment and physical support are essential for improving their self-rated health status.

IJPHS Vol. 3, No. 2, June 2014: $69-80$ 


\section{ACKNOWLEDGEMENTS}

The present study was prepared using a dataset from the National Center for Health Statistics. The findings and conclusion do not necessarily represent the opinions or views of the National Center for Health Statistics. The author also thank Dr. Eryn Murphy for her through review.

\section{REFERENCES}

[1] U.S. Census Bureau, "Facts for Features: Elder Americans Month", 2011. Available at: http://www.census.gov/newsroom/releases/archives/facts_for_features_special_editions/cb11-ff08.html.

[2] U.S. Census Bureau, "The U.S. Census Bureau Reports World's Elder Population Projected to Triple by 2050", 2009. Available at: http://www.census.gov/newsroom/releases/archives/international_population/cb09-97.html.

[3] Mossey J. M., Shapiro E., "Self-rated health: a predictor of mortality among the elderly", Am J Public Health, vol/issue: 72(8), pp. 800-808, 1982.

[4] DeSalvo K. B., Fan V. S., McDonell M. B., Fihn S. D., "Predicting mortality and healthcare utilization with a single question”, Health Serv Res, vol./issue: 40(4), pp. 1234-1246, 2005.

[5] Blazer D.G., "How do you feel about...? Health outcomes in late life and self-perceptions of health and wellbeing”, Gerontologist, vol/issue: 48(4), pp. 415-422, 2008.

[6] Desalvo K. B., Muntner P., "Discordance between physician and patient self-rated health and all-cause mortality", Ochsner J, vol/issue: 11(3), pp. 232-240, 2011.

[7] Dominick K. L., Ahern F. M., Gold C. H., Heller D. A., "Relationship of health-related quality of life to health care utilization and mortality among elder adults”, Aging ClinExp Res, vol/issue: 14(6), pp. 499-508, 2002.

[8] Ettner S. L., "New evidence on the relationship between income and health”, J Health Econ, vol/issue: 15(1), pp. 67-85, 1996.

[9] Kennedy B. P., Kawachi I., Glass R., Prothrow-Stith D., "Income distribution, socioeconomic status, and self-rated health in the United States: multilevel analysis”, BMJ, vol/issue: 317(7163), pp. 917-921, 1998.

[10] Ferraro K. F., Kelley-Moore J. A., "Self-rated health and mortality among black and white adults: examining the dynamic evaluation thesis”, J Gerontol B PsycholSciSocSci, vol/issue: 56(4), pp. S915-205, 2001.

[11] Zajacova A., Dowd J. B., "Reliability of Self-rated Health in US Adults”, Am J Epidemiol, vol/issue: 174(8), pp. 977-983, 2011.

[12] NCHS, "LSOA II. The Second Longitudinal Study of Aging", $2009 . \quad$ Available at:http://www.cdc.gov/nchs/r\&d/nchs_datalinkage/lsoaii_data_linkage_mortality_activities.htm.

[13] Liu L., Newschaffer C. J., "Impact of social connections on risk of heart disease, cancer, and all-cause mortality among elderly Americans: findings from the Second Longitudinal Study of Aging (LSOA II)", Arch Gerontol Geriatr, vol/issue: 53(2), pp. 168-173, 2011.

[14] Johnson R. J., Wolinsky F. D., "The structure of health status among elder adults: disease, disability, functional limitation, and perceived health”, J Health SocBehav, vol/issue: 34(2), pp. 105-121, 1993.

[15] Subramanian S. V., Kim D., Kawachi I., "Covariation in the socioeconomic determinants of self rated health and happiness: a multivariate multilevel analysis of individuals and communities in the US”, $J$ Epidemiol Community Health, vol/issue: 59(8), pp. 664-669, 2005.

[16] Breidablik H. J., Meland E., Lydersen S., "Self-rated health during adolescence: stability and predictors of change (Young-HUNT study, Norway)”, Eur J Public Health, vol/issue: 19(1), pp. 73-78, 2009.

[17] Shadbolt B., "Some correlates of self-rated health for Australian women”, Am J Public Health, vol/issue: 87(6), pp. 951-956, 1997.

[18] Lundberg O., Manderbacka K., “Assessing reliability of a measure of self-rated health”, Scand J Soc Med, vol/issue: 24(3), pp. 218-224, 1996

[19] Crossley T. F., Kennedy S., “The reliability of self-assessed health status”, J Health Econ, vol/issue: 21(4), pp. 643-658, 2002.

[20] Smith J. P., "Healthy bodies and thick wallets: the dual relation between health and economic status", $J$ Econ Perspect, vol/issue: 13(2), pp. 144-166, 1999.

[21] Schüz B., Wurm S., Schöllgen I., Tesch-Römer C., "What do people include when they self-rate their health? Differential associations according to health status in community-dwelling elder adults", Qual Life Res, vol/issue: 20(10), pp. 1573-1580, 2011.

[22] Leinonen R., Heikkinen E., Jylhä M., "Changes in health, functional performance and activity predict changes in self-rated health: a 10-year follow-up study in elder people”, Arch GerontolGeriatr, vol/issue: 35(1), pp. 79-92, 2002.

[23] Leskinen R., Antikainen R., Peltonen M., Sippola R., Jousilahti P., Laatikainen T., "Determinants of changes in self-rated health among Finnish war veterans: Results from the Veteran Project 1992 and 2004 surveys", Arch GerontolGeriatr, vol/issue: 55(2), pp. 343-348, 2011.

[24] Boyce W. F., Davies D., Raman S. R., Tynjälä J., Välimaa R., King M., Gallupe O., Kannas L., "Emotional health of Canadian and Finnish students with disabilities or chronic conditions”, Int J Rehabil Res, vol/issue: 32(2), pp. 154-161, 2009. 
[25] Blackman J. A., Gurka M. J., Gurka K. K., Oliver M. N., "Emotional, Developmental and Behavioural CoMorbidities of Children with Chronic Health Conditions", Journal of paediatrics and child health, vol/issue: 47(10), pp. 742-747, 2011.

[26] Han B., Phillips C., Ferrucci L., Bandeen-Roche K., Jylha M., Kasper J., Guralnik J. M., “Change in self-rated health and mortality among community-dwelling disabled elder women”, Gerontologist, vol/issue: 45(2), pp.216221, 2005. 